ISSN 0103-5150

Fisioter. Mov., Curitiba, v. 24, n. 1, p. 99-106, jan./mar. 2011 Licenciado sob uma Licença Creative Commons

\title{
Mobilidade articular de idosos diabéticos e não diabéticos e influência da fisioterapia
}

\author{
Joint mobility of diabetic and non-diabetic elderly people and \\ influence of the physical therapy
}

\section{Lorena Soares Ulhoa ${ }^{[a]}$, Rafael Chedid Oliveira Lima ${ }^{[b]}$, Verusca Najara de Carvalho Cunha ${ }^{[c]}$, Erika Baptista Gomes ${ }^{[\mathrm{d}]}$, Carmen Sílvia Grubert Campbell ${ }^{[\mathrm{e}]}$, Hermelinda Cordeiro Pedrosa ${ }^{[\mathrm{f}]}$}

[a] Fisioterapeuta pela Universidade Católica de Brasília (UCB), Graduada em Educação Física pela Universidade de Brasília (UnB), Brasília, DF - Brasil, e-mail: loresulhoa@gmail.com

[b] Fisioterapeuta pela Universidade Católica de Brasília (UCB), Brasília, DF - Brasil, e-mail: wernestch@hotmail.com

[c] Fisioterapeuta pela Universidade Católica de Brasília (UCB), Mestranda em Educação Física pela Universidade Católica de Brasília (UCB), Brasília, DF - Brasil, email: najavrusk@gmail.com

[d] Fisioterapeuta, Mestre em Ciências Médicas pela Universidade de Brasília (UnB), supervisora de estágio nas áreas de Neurologia na Universidade Católica de Brasília (UCB), Brasília, DF - Brasil, e-mail: erika@ucb.br

[e] Educadora física, Mestre em Ciência da Motricidade pela Universidade Estadual Paulista Júlio de Mesquita Filho (UNESP), Doutora em Ciências (Fisiologia Humana) pela Universidade de São Paulo (USP), docente do Programa de Mestrado e Doutorado em Educação Física da Universidade Católica de Brasília (UCB), Brasília, DF - Brasil, e-mail: campbellcsg@gmail.com

[f] Endocrinologista, coordenadora do Programa de Diabetes do Hospital Regional de Taguatinga, Brasília, DF - Brasil, e-mail: pedrosalinda@terra.com.br

\section{Resumo}

Introdução: 0 diabetes tipo 2 (DM) é uma doença crônica que tem impacto socioeconômico importante nos dias atuais. Medidas de prevenção, detecção precoce e tratamento se mostram importantes para a diminuição da taxa de morbimortalidade dessa síndrome. Objetivos: Verificar e comparar entre idosos, portadores ou não de diabetes mellitus, os que possuem maior limitação de mobilidade articular (LMA); analisar a relação da LMA com a idade, o tempo de DM, a presença de sinal da prece (SP), o risco neuropático (RN) e a influência dos exercícios utilizados na fisioterapia. Materiais e métodos: Os indivíduos foram divididos em três grupos com $n=15$ cada, sendo grupo C (controle), grupo DM, grupo DMF (DM em tratamento fisioterapêutico, por meio de alongamentos e exercícios leves). Foram avaliadas as goniometrias de tornozelos 
e punhos, $\mathrm{SP}$ e RN, bem como relacionou-se a média das amplitudes de movimento (ADMs) entre os grupos com a idade, o tempo de DM, o SP e o RN. Resultados e conclusões: A média das ADMs nos grupos foram $\mathrm{C}>\mathrm{DMF}>\mathrm{DM}$, de forma significativa $(\mathrm{p}<0,05)$, exceto flexão de punho direito. Observa-se a influência da fisioterapia (DMF > DM), significante no movimento de extensão de punho. Quanto maior a idade e o tempo de DM maior a tendência de LMA, porém, essas diferenças não foram significativas. No presente estudo, o RN e o SP não são os únicos determinantes da LMA. Conclui-se que o diabetes interfere significativamente na ADM e que houve influência benéfica da fisioterapia, por meio de alongamentos e exercícios leves.

Palavras-chave: Limitação da mobilidade articular. Diabetes mellitus. Idosos.

\section{Abstract}

Introduction: Type 2 diabetes (T2D) is a chronical disease with an important socioeconomic impact nowadays. Prevention, early detection and treatment are important to morbidity and mortality reduction. Objectives: To verify and compare the which have the greatest limitation of joint mobility (LJM), the LJM relation to age, the time of diagnosis of T2D, the presence of a prayer signal (PS), the risk neuropathic (RN) and the influence of exercises used in physiotherapy in elderly T2D and non T2D individuals. Materials and methods: The subjects were accomplished in three groups being control group ( $C ; n=15)$; T2D group (T2D; $n=15)$ and T2D in physiotherapeutic treatment - stretching and light resistance exercises (T2DPhys; $n=15)$. Goniometry of the ankles and wrists, PS and RN were evaluated and the average of range of motion (ROM) was associated between groups accordingly to the age, time of diagnosis of T2D, PS and RN. Results and conclusions: The average ROM in groups were $C>T 2 D P h y s>T 2 D$, significantly $(p<0.05)$, except for right wrist flexion. There was a significant $(p<0.05)$ influence of physiotherapy (T2DPhys $>$ T2D)in the wrist extension movement. The LJM were higher in older individual and in T2S with more time with disease but these relations were not significant. In this study, the RN and PS were not the only determinants of LJM. It was concluded that T2D interferes negatively and significantly in ROM and the physiotherapy treatment through stretching and resistance light exercises exerted beneficial influences on these parameters in the T2D.

Keywords: Limitation of articular mobility. Diabetes mellitus. Elderly.

\section{Introdução}

As complicações do diabetes mellitus (DM) têm um impacto socioeconômico significativo, não só para o indivíduo diabético, mas também para a sociedade. Estratégias de manejo privilegiam a prevenção, a detecção precoce e o tratamento apropriado do DM e de suas complicações, para a diminuição da morbidade e da mortalidade dos diabéticos (1).

Uma complicação crônica bastante comum é a limitação da mobilidade articular (LMA), demonstrada inicialmente nas mãos, classificada pelo sinal da prece $(2,3)$, mas que também pode acometer coluna, joelhos, quadril, tornozelos, punhos e cotovelos $(2,4-7)$. A prevalência da LMA, em indivíduos com DM, tem oscilado entre $8 \%$ e $58 \%$. Essa variação ocorre em virtude das diferenças na população estudada e da forma como é realizada a avaliação $(4,5,8,9)$.
0 risco da LMA em diabéticos aumenta com os valores da hemoglobina glicada (A1c), com a duração da doença e com a idade do indivíduo $(1,4,8,10$, 11). Torna-se, portanto, relevante a sua detecção, pois ela pode causar maiores prejuízos ao diabético, principalmente quando a articulação do tornozelo é comprometida por causa de fatores como aumento da rigidez, atrofia da musculatura e alteração na estrutura do colágeno, gerando hiperpressões plantares, o que acarreta alterações de equilíbrio, de marcha e, de forma mais grave, pode ocasionar ulcerações quando associada à neuropatia diabética $(2,12-14)$.

0 presente estudo teve como objetivo comparar, em indivíduos entre 61 e 75 anos, portadores ou não de DM, a limitação da mobilidade articular, além de verificar a relação da mobilidade articular com a idade, o tempo de DM, a presença de sinal da prece (SP), o risco neuropático (RN) e se há interferência 
do tratamento fisioterapêutico na mobilidade articular entre os indivíduos diabéticos.

\section{Materiais e métodos}

A amostra foi composta por 45 idosos, sendo 17 indivíduos do sexo masculino e 28 do sexo feminino, na faixa etária entre 61 e 75 anos. Os participantes foram divididos em 3 grupos, cada um com 15 integrantes: grupo C (controle), constituído por indivíduos não diabéticos e que não participavam de um programa regular de exercício físico; grupo DM, formado por indivíduos portadores de DM e que também não realizavam um programa regular de atividade física; e grupo DMF, constituído por indivíduos diabéticos que estavam realizando tratamento fisioterapêutico por meio de alongamentos e exercícios leves (flexão/extensão e abdução/ adução de membros superiores e inferiores além de movimentos de tronco), realizados com auxílio de bastões e halteres. Os exercícios eram realizados no ambulatório de diabetes e no Hospital Regional.

Foram excluídos do estudo dois indivíduos que se enquadraram em pelo menos um dos seguintes critérios: sequela neurológica; história de trauma prévio nos últimos seis meses - fratura, entorses, luxação, tendinite; problema reumatológico; deformidades congênitas e ou adquiridas de outras causas além do DM.

Após aprovação pelo Comitê de Ética e Pesquisa, de acordo com a Resolução n. 196/96, capítulo IX.2, todos os participantes assinaram um Termo de Consentimento Livre e Esclarecido (TCLE). Os dados coletados foram registrados em uma ficha de avaliação, em que foi verificada a presença ou não do SP e do $\mathrm{RN}$ e realizada goniometria passiva, utilizando-se goniômetro universal (Carci Brasil) para a avaliação das articulações do tornozelo (flexão plantar e dorsiflexão) e punho (flexão e extensão). Um único avaliador, após treinamento, realizou essas medidas no Ambulatório de Diabetes e no Hospital Regional.

A avaliação do SP foi realizada pedindo-se para o indivíduo unir as palmas das mãos, na posição de oração (8). 0 indivíduo foi classificado com sinal presente quando não conseguia unir perfeitamente as superfícies palmares, por conta das limitações das articulações interfalangeanas, segundo Gomes (12).

Para a verificação do risco neuropático foi utilizado o monofilamento de $10 \mathrm{~g}$ do kit estesiômetro
Semmes-Weinstein (SORRI-Brasil) no 1ํㅡㄹ no $3^{\circ}$ e no 5 o pododáctilo e metatarso (15). 0 paciente foi testado em decúbito dorsal. Inicialmente, foi realizada uma aplicação na mão para que este reconhecesse o teste. Em seguida, com o paciente com os olhos fechados, pediu-se que relatasse quando sentisse a pressão do monofilamento e o local dessa aplicação. 0 teste foi padronizado com duas aplicações em cada local, sendo a segunda descartada caso percebesse a primeira. A pressão foi realizada por dois segundos com o estesiômetro em posição perpendicular à superfície testada, sendo a ordem das aplicações randomizada pelo examinador. Foi considerado RN quando o paciente não conseguia identificar pelo menos um dos pontos de aplicação $(16,17)$.

A goniometria de punho foi padronizada mantendo-se o antebraço e a mão em pronação. 0 goniômetro foi centrado no processo estiloide ulnar, com o braço fixo do goniômetro paralelo ao eixo longitudinal do antebraço ao longo da borda ulnar, e o braço móvel paralelo ao eixo longitudinal do quinto metacárpico, sendo este movido para medir a flexão e a extensão. Na avaliação do tornozelo foi estipulado que o indivíduo permanecesse sentado com o joelho fletido em um ângulo de $90^{\circ}$ sobre a beira da maca e com a face plantar dos pés pendentes e paralelas ao solo. 0 braço fixo foi colocado sobre uma linha paralela ao eixo longitudinal da fíbula na face lateral da perna, o centro do goniômetro foi colocado na projeção do maléolo na borda lateral do pé alinhado com o eixo longitudinal da fíbula. 0 braço móvel foi posto paralelo ao eixo longitudinal do quinto metatársico, realizando-se o movimento de dorsiflexão e flexão plantar e verificando-se, assim, a amplitude dos movimentos descritos (18).

\section{Análise estatística}

Foi feita análise estatística descritiva dos três grupos, seguida pelo teste de análise de variância (ANOVA), para testar a hipótese nula de igualdade entre os grupos em relação à amplitude articular, considerando $p$-valor $<0,05$. Com o objetivo de se comparar o grupo DM e DMF com o grupo controle, realizou-se o teste de Dunnett e, para comparar o grupo DM com o DMF, realizou-se o teste de Duncan.

O coeficiente de correlação de Pearson foi utilizado para relacionar a idade e o tempo de diabetes com a mobilidade articular, e para se avaliar a 
relação entre sinal da prece e risco neuropático com a mobilidade articular utilizou-se o Box-Plot.

\section{Resultados}

A Tabela 1 apresenta os dados de descrição dos grupos quanto à idade, ao sexo, ao tempo de diabetes e à presença de RN e SP.

Tabela 1 - Dados demográficos da população em estudo

\begin{tabular}{lccc}
\hline & Grupo C & Grupo DM & Grupo DMF \\
\hline Idade (anos) & $68,7 \pm 5,8$ & $63,6 \pm 3,0$ & $68,0 \pm 5,3$ \\
Sexo (f/m) & $10 / 5$ & $9 / 6$ & $9 / 6$ \\
TD (anos) & & $14,1 \pm 10,4$ & $13,5 \pm 9,6$ \\
RN (\%) & & 26,7 & 0 \\
SP (\%) & & 60 & 46,60 \\
\hline
\end{tabular}

Obs.: todos os valores são expressos por médias \pm DP ou por frequência (\%).

Legenda: TD = tempo de diabetes diagnosticado; $\mathrm{RN}=$ risco neuropático; SP = sinal da prece.
Ao analisar a média de amplitude articular dos movimentos de punho e tornozelo, o grupo C (controle) obteve maior média, seguido pelos grupos DMF e DM, consecutivamente (Tabelas 2 e 3). Por meio do teste de variância ANOVA, pode-se verificar que todas as médias das amplitudes dos movimentos de tornozelo e punho, exceto flexão direita, foram significativamente menores nos grupos DM e DMF quando comparados ao grupo C $(\mathrm{p}<0,05)$.

Comparando-se o grupo controle com o grupo DM, em relação ao punho, verificou-se significância nas médias dos movimentos de flexão esquerda (FE), extensão direita (ED) e esquerda (EE); na comparação do grupo controle com o grupo DMF houve significância apenas no movimento EE; e no grupo DM com o DMF, significância em ED e EE.

Quanto às médias das amplitudes de tornozelo (Tabela 3), ao comparar o grupo C com o DM houve diferença estatisticamente significante em todos os movimentos articulares, não ocorrendo o mesmo para as comparações entre o grupo $\mathrm{C}$ com o DMF, onde somente não houve diferença significante para o movimento de flexão plantar esquerda. Não se verificou diferença entre os parâmetros dos grupos DM e DMF.

Tabela 2 - Média \pm DP da amplitude articular em graus da articulação de punho e significância

\begin{tabular}{lllccccc}
\hline & Grupo C & Grupo DM & Grupo DMF & P (C/DM/DMF) & Dnt (C/DM) & Dnt (C/DMF) & Dnc (DM/DMF) \\
\hline FD & $84,7 \pm 4,4$ & $79,0 \pm 10,6$ & $81,2 \pm 8,3$ & 0,1792 & & & \\
FE & $85,5 \pm 4,4$ & $77,3 \pm 8,4$ & $82,2 \pm 6,7$ & 0,0073 & $*$ & & \\
ED & $74,9 \pm 7,0$ & $58,3 \pm 10,8$ & $68,8 \pm 9,8$ & 0,0001 & $*$ & & $*$ \\
EE & $76,9 \pm 5,0$ & $56,1 \pm 9,6$ & $69,4 \pm 7,0$ & 0,0001 & $*$ & $*$ & $*$ \\
\hline
\end{tabular}

Legenda: $F D=$ flexão direita; $F E$ = flexão esquerda; $E D=$ extensão direita; $E E$ = extensão esquerda; $P(C / D M / D M F)=$ teste estatístico ANOVA, p-valor < 0,05; Dnt (C/DM) e Dnt (C/DMF) = teste estatístico de Dunnett; Dnc (DM/DMF) = teste estatístico de Duncan; $\star=p<0,05$.

Tabela 3 - Média \pm DP da amplitude articular do tornozelo

\begin{tabular}{lccccccc}
\hline & Grupo C & Grupo DM & Grupo DMF & P (C/DM/DMF) & Dnt (C/DM) & Dnt (C/DMF) & Dnc (DM/DMF) \\
\hline FPD & $28,5 \pm 2,9$ & $22,8 \pm 4,4$ & $23,5 \pm 6,2$ & 0,0033 & $*$ & $*$ & \\
FPE & $28,5 \pm 3,5$ & $23,3 \pm 6,5$ & $25,0 \pm 6,3$ & 0,0456 & $*$ & & \\
DD & $17,3 \pm 3,5$ & $10,7 \pm 3,4$ & $12,7 \pm 4,2$ & 0,0001 & $*$ & $*$ & \\
DE & $17,7 \pm 2,8$ & $11,4 \pm 4,2$ & $13,1 \pm 4,3$ & 0,0002 & $*$ & $*$ & \\
\hline
\end{tabular}

Legenda: FPD = flexão plantar direita; FPE = flexão plantar esquerda; $D D=$ dorsiflexão direita; $D E$ = dorsiflexão esquerda; $P(C / D M / D M F)=$ teste estatístico ANOVA, p-valor < 0,05; Dnt (C/DM) e Dnt (C/DMF) = teste estatístico de Dunnett; Dnc (DM/DMF) = teste estatístico de Duncan; ${ }^{*}=p<0,05$. 
Relacionando-se a média das amplitudes articulares com os valores normais, tomando como base Knutzen $(19,20)$ (Tabela 4), verifica-se que no movimento de flexão de punho os três grupos obtiveram valores dentro da faixa de normalidade e, no movimento de extensão, apenas o grupo controle obteve amplitude normal. Com relação aos movimentos de flexão plantar e dorsiflexão, os três grupos encontraram-se com a amplitude reduzida.

Tabela 4 - Valores normais para a amplitude articular de punho e tornozelo

\begin{tabular}{cc}
\hline Movimento & Valor normal \\
\hline Flexão de punho & $70-90^{\circ}$ \\
Extensão de punho & $70-80^{\circ}$ \\
Dorsiflexão & $20^{\circ}$ \\
Flexão plantar & $50^{\circ}$ \\
\hline
\end{tabular}

Fonte: Knutzen, 1999.

Por meio do coeficiente de Pearson, verificou-se que quanto maior a idade há uma tendência na redução da mobilidade dentro dos grupos C e DM, porém, esses resultados não foram significativos $(\mathrm{p}>$ $0,05)$, ou seja, não se pode inferir que a mobilidade está relacionada com a idade. 0 mesmo resultado foi encontrado na relação entre o tempo de diabetes e a mobilidade articular, ou seja, quanto maior o tempo de diabetes há uma redução da mobilidade, porém, esses resultados também não foram significativos.

Tanto o RN quanto o SP não podem ser diretamente relacionados com a mobilidade articular, pois indivíduos que não possuíam RN e/ou SP apresentaram diminuição da mobilidade articular.

\section{Discussão}

A detecção da LMA envolve o uso de diversas técnicas, tal como o método do sinal da prece, sendo este um indicativo de LMA para qualquer articulação (21). No presente estudo, verificou-se que indivíduos que não possuíam SP apresentavam LMA em pelo menos uma articulação, ou seja, apenas esse sinal não é indicativo de LMA. Uma das investigações mais recentes, entretanto, utiliza a goniometria para comparar as diferenças entre uma população diabética e não diabética $(18,21)$, ou seja, o goniômetro, por ser um instrumento quantitativo, vem sendo cada vez mais utilizado, tornando-se utensílio indispensável para a mensuração da LMA e a classificação da severidade desta.

Por meio dos resultados das médias das amplitudes articulares é possível verificar que, com exceção da FD, há uma diferença significativa entre os grupos C, DM e DMF. No estudo de Pal et al. (22), 23\% do controle, indivíduos não diabéticos, apresentava achados clínicos de LMA. Com isso, foi levantada a hipótese de que a LMA está relacionada primariamente a um processo natural de envelhecimento, graças a processos degenerativos da velhice e à redução da atividade física. No presente estudo, verificou-se que o grupo controle apresentou LMA, principalmente de tornozelo, mas ainda assim com amplitudes maiores do que o grupo de diabéticos (DM e DMF), e que nos diabéticos que faziam fisioterapia a mobilidade articular era maior que no grupo que não realizava.

0 risco da LMA em diabéticos aumenta com a duração da doença e com a idade do indivíduo, porém, neste trabalho foi possível observar que, apesar dessa tendência, os resultados não foram significativos $(4,8,10,21)$.

Observa-se que, apesar dos indivíduos dos três grupos estarem numa mesma faixa etária, o fator diabetes interferiu de forma significativa na redução da mobilidade articular. Comparando-se o grupo C com o DM, em que só o fator diabetes é o diferencial entre os dois grupos, verifica-se que há uma diferença significativa, com exceção da FD. Comparando o grupo C com o DMF foram significativos EE, FPD, DD e DE.

Existem diversas teorias que explicam a LMA em participantes diabéticos, sendo estas: glicosilação não enzimática (GNE), hidratação aumentada de colágeno, aumento da ligação cruzada de proteínas, edema do tecido conjuntivo por meio da via aldoseredutase, microangiopatias e neuropatias $(4,5,8)$.

0 colágeno é uma proteína que dá origem à maioria dos tecidos e órgãos do corpo. Existem pelo menos 18 tipos de colágeno, sendo o tipo I o que está presente na pele, nos tendões, nos ossos, nos ligamentos e nas fáscias. Com o avançar da idade ou a presença de doenças como o DM, o colágeno sofre processos degenerativos irreversíveis. Acredita-se que o diabético e o indivíduo mais idoso sofram as 
mesmas alterações no colágeno, porém, no diabético isso ocorreria em uma faixa etária mais precoce, pela alteração metabólica e pelos danos causados pela falta de controle glicêmico (23). Pode-se observar isso por meio das médias das idades dos três grupos. 0 grupo constituído por diabéticos que não realizavam fisioterapia ou um programa de exercício físico (Grupo DM) possuía uma média menor com relação à idade, porém, foi o grupo que apresentou maior limitação da mobilidade articular.

Outra explicação para a LMA é a glicosilação não enzimática (GNE) de algumas proteínas do corpo, gerada por uma hiperglicemia tecidual persistente, levando a alterações das propriedades mecânicas dos tecidos, geralmente reduzindo a elasticidade (24). Isso acontece por conta da forte interação dos grupos aldeído e cetona dos açúcares biológicos com os grupos amino livres do colágeno, fazendo com que este sofra glicosilação irreversível e se acumule em grandes quantidades no tecido conjuntivo $(5,8,23)$.

Outra possível explicação é o edema hidrofílico do tecido conjuntivo causado por alterações do colágeno (hidratação aumentada), o que acarreta progressivo espessamento dérmico diminuindo a mobilidade articular $(5,8)$.

A ligação cruzada do colágeno influencia a resistência, a meia-vida e a solubilidade deste. Com o aumento dessas ligações, a colagenase, enzima responsável pela degradação do colágeno, não será efetiva, gerando maior acúmulo de colágeno na derme dos participantes diabéticos $(5,8)$.

Outra enzima bastante encontrada no tecido conjuntivo é a aldose-redutase, que possui grande afinidade com a glicose. Com a hiperglicemia persistente, há uma maior ação da aldose-redutase, levando à superprodução de sorbitol e a outros polióis, que saturam a via da enzima sorbitol-deidrogenase acumulando-se no meio intracelular. Graças a esse gradiente osmótico há uma tendência para a entrada de água no interior da célula, levando ao edema celular, ao espessamento cutâneo e à rigidez de estruturas periarticulares $(5,8)$.

De acordo com Islabao et al. (5), acredita-se que a microangiopatia acarrete LMA em virtude de um processo de hipóxia local, aumentando a resposta primária do fibroblasto que, consequentemente, aumentaria a produção de colágeno.

As neuropatias periféricas podem, também, acarretar LMA pela possibilidade destas levarem a um desuso do membro $(5,25)$. No presente estudo, ve- rificou-se que mesmo indivíduos que não apresentaram RN tinham LMA, daí a importância de estar sempre utilizando a avaliação articular.

Toda redução funcional pode contribuir para a diminuição da qualidade de vida. A prevenção é um dos principais aspectos a ser orientado com o objetivo de evitar maiores complicações, segundo Sacco et al. (26).

Embora não exista nenhuma intervenção direta sobre a LMA, alguns autores $(8,11)$ preconizam o controle glicêmico como forma de minimizar essas complicações e, segundo Bowker e Pfeifer (24), existem certos medicamentos que podem ajudar a controlar a deposição de colágeno, como, por exemplo, a n-fenaciltiazólio, que cliva as ligações cruzadas do colágeno, e a penicilina, que inibe a ligação cruzada.

A fisioterapia pode, também, por meio de seus recursos, melhorar o controle glicêmico, prevenir a LMA e melhorar a função desses participantes. Curran et al., citados por Bowker e Pfeifer (24), foram os primeiros a demonstrar que um programa de fisioterapia poderia ser eficaz para melhorar a mobilidade articular dos participantes diabéticos com LMA. Neste estudo, foi possível verificar a influência da fisioterapia, pois a média das amplitudes articulares no grupo de diabéticos que realizavam fisioterapia foi maior que no grupo que não realizava, com valores significativos nos movimentos de extensão de punho para a direita e a esquerda.

\section{Conclusão}

Pode-se concluir que idosos diabéticos possuem menor mobilidade articular quando comparados a um grupo da mesma faixa etária, porém, sem a patologia. Apesar da amplitude articular diminuir com o aumento da idade e o tempo de diabetes, não se pode inferir que possuam uma relação direta com a LMA ( $p>0,05)$. O SP e o RN não são os únicos determinantes da LMA, pois $100 \%$ dos indivíduos avaliados possuíam LMA pelo menos em alguma das articulações testadas, com base em Knutzen $(19,20)$, ou seja, mesmo com a ausência do SP e do RN apresentaram diminuição da mobilidade articular.

Uma dificuldade encontrada no presente trabalho foi a de se obter um valor padrão de mobilidade articular relacionada com o envelhecimento. Dessa forma, sugere-se que sejam realizadas novas 
pesquisas para se obter valores padrão de acordo com a faixa etária.

Verifica-se a importância da fisioterapia na abordagem desse paciente, sendo relevante a realização de novos estudos envolvendo avaliação goniométrica pré e pós-tratamento fisioterapêutico protocolado para comprovar e quantificar a eficácia deste.

\section{Agradecimentos}

Agradecemos a colaboração de cada participante voluntário do trabalho, o Departamento de Fisioterapia da Universidade, o Hospital Regional, pelo apoio prestado a nossa pesquisa, e a Daniele B. de Oliveira pela sua participação neste estudo.

\section{Referências}

1. International Diabetes Federation. Complicações do diabetes e educação. Diabetes Clínica. 2002;6(3): 217-20.

2. Férnando DJS, Masson EA, Veves A, Boulton AJM. Relationship of limited joint mobility to abnormal foot pressures and diabetic foot ulceration. Diabetes Care. 1991;14(1):8-11.

3. Fernando DJS, Vernidharan J. Limited joint mobility in Sri Lankan patients with non-insulin-dependent diabetes. Br J Rheumatol. 1997;36(3):374-6.

4. Arkkila PET, Gautier JF. Musculoskeletal disorders in diabetes mellitus: an update. Best Pract Res Clin Rheumatol. 2003;17(6):945-70.

5. Islabao AG, Pinto MEB, Groboropatel D, Gracia FO, Staub HL, Keiserman MW. Síndrome da mobilidade articular limitada: novos aspectos no diabético. Rev Bras Reumatol. 1994;34(1):38-43.

6. Simmons RW, Richardson C, Deutsch K. Limited joint mobility of the ankle in diabetic patients with cutaneous sensory deficit. Diabetes Res Clin Pract. 1997;37(2):137-43.

7. Savas S, Köroglu BK, Koyuncuoglu HR, Uzarc E, Çelika H, Tameret NM. The effects of the diabetes related soft tissue hand lesions and the reduced hand strength on functional disability of hand in type 2 diabetic patients. Diabetes Res Clin Pract. 2007;77(1): 77-83.
8. Wright V, Hordon LD. Limited joint in diabetes mellitus [CD-ROM]. UpToDate ${ }^{\circledR} 1998$.

9. Lindsay JR, Kennedy L, Atkinson AB, Bell PM, Carson DJ, McCance DR, et al. Reduced prevalence of limited joint mobility in type I diabetes in a UK clinic population over a 20-year period. Diabetes Care. 2005; 28(3):658-61.

10. Aoki Y, Yazaki K, Shirotori K, Yanagisawa Y, Oguchi H, Kiyosawa K, et al. Stiffening of connective tissue in elderly diabetic patients: relevance to diabetic nephropathy and oxidative stress. Diabetologia. 1993; 36(1):79-83.

11. Tesfaye S, Kempler P. Painful diabetic neuropathy. Diabetologia. 2005;48(5):805-7.

12. Gomes EB. Avaliação e diagnóstico da pressão plantar em participantes diabéticos com e sem neuropatia diabética distal: aplicação dos testes semiquantitativos utilizando os plantígrafos Harris Mat e Pressure Stat [dissertação]. Brasília: Universidade Católica de Brasília; 2004.

13. Sacco ICN, João SMA, Alignani D, Ota DK, Sartor CDS, Silveira LT, et al. Implementing a clinical assessment protocol for sensory and skeletal function in diabetic neuropathy patients at a university hospital in Brazil. São Paulo Med J. 2005;123(5):229-33.

14. Viswanathan V, Snehalatha C, Sivagami M, Seena R, Ramachandran A. Association of limited joint mobility and high plantar pressure in diabetic foot ulceration in Asian Indians. Diabetes Res Clin Pract. 2003;60(1):57-61.

15. Sociedade Brasileira de Diabetes. Consenso brasileiro de conceitos e condutas para o diabetes mellitus - recomendações da Sociedade Brasileira de Diabetes para a prática clínica. São Paulo: Sociedade Brasileira de Diabetes; 1997.

16. Grupo de Trabalho Sobre Pé Diabético. Consenso internacional sobre pé diabético. Brasília: Secr Est Saúde; 2001.

17. Pitta GBB, Castro AA, Burihan E. Angiologia e cirurgia vascular: guia ilustrado. [acesso em 2 jan. 2009]. Disponível em: http://www.lava.med.br/livro

18. Cole TM, Tobis JS. Medição da função musculoesquelética. In: Kottke FJ, Lehmann JF. Tratado de medicina física e reabilitação. São Paulo: Manole; 1994. p. 19-30. 
19. Knutzen KM, Hamill J. Anatomia funcional do membro inferior. In: Knutzen KM, Hamill J. Bases biomecânicas do movimento humano. São Paulo: Manole; 1999. p. 202-67.

20. Knutzen KM, Hamill, J. Anatomia funcional do membro superior. In: Knutzen KM, Hamill J. Bases biomecânicas do movimento humano. São Paulo: Manole; 1999. p. 146-89.

21. Saunseng S, Kastenbauer T, Irsigler K. Limited joint mobility in selected hand and foot joints in patients with type 1 diabetes mellitus: a methodology comparison. Diabetes Nutr Metab. 2002;15(1):1-6.

22. Pal B, Anderson J, Dick WC, Griffiths ID. Limitation of joint mobility and shoulder capsulits in insulin and non-insulin-dependent diabetes mellitus. Br J Rheumatol. 1986;25(2):147-51.

23. Bentley J. Collagen and development of diabetic foot complications. The Diabetic Foot. 2001;4(4):194-9.
24. Bowker JH, Pfeifer MA. Levin e O'Neal: 0 pé diabético. 6a ed. Rio de Janeiro: Dilivros; 2002.

25. Rosenbloom AL, Silverstein JH, Lezotte DC, Richardson KB, McCallum MR. Limited joint mobility in childhood diabetes mellitus indicates increased risk for microvascular disease. N Engl J Med. 1981;305(4):191-4.

26. Sacco ICN, Sartor CD, Gomes AA, João SMA, Cronfli R. Assessment of motor sensory losses in the foot and ankle due to diabetic neuropathy. Rev Bras Fisioter. 2007;11(1):27-33.

Recebido: 13/07/2010

Received: 07/13/2010

Aprovado: 10/11/2010

Approved: 11/10/2010 